\title{
Radiation Therapy Alone in cT1-3NO Non-small Cell Lung Cancer Patients Who Are Unfit for Surgical Resection or Stereotactic Radiation Therapy: Comparison of Risk-Adaptive Dose Schedules
}

\author{
Won Kyung Cho, MD \\ Jae Myoung Noh, MD \\ Yong Chan Ahn, MD, PhD \\ Dongryul Oh, MD \\ Hongryull Pyo, MD, PhD
}

Department of Radiation Oncology,

Samsung Medical Center,

Sungkyunkwan University

School of Medicine, Seoul, Korea

\begin{abstract}
Purpose
High dose definitive radiation therapy (RT) alone is recommended to patients with cT1-3NO non-small cell lung cancer, who are unfit for surgery or stereotactic RT. This study was conducted to evaluate the clinical outcomes and cost-effectiveness following RT alone using two different modest hypofractionation dose schemes.
\end{abstract}

\section{Materials and Methods}

Between 2001 and 2014, 124 patients underwent RT alone. From 2001 till 2010, 60 Gy in 20 fractions was delivered to 79 patients (group 1). Since 2011, 60 Gy in 20 fractions (group 2, 20 patients), and 60 Gy in 15 fractions (group 3, 25 patients) were selectively chosen depending on estimated risk of esophagitis.

\section{Results}

At follow-up of 16.7 months, 2-year rates of local control, progression-free survival, and overall survival were $62.6 \%, 39.1 \%$, and $59.1 \%$, respectively. Overall survival was significantly better in group $3(\mathrm{p}=0.002)$. In multivariate analyses, cT3 was the most powerful adverse factor affecting clinical outcomes. Incidence and severity of radiation pneumonitis were not different among groups, while no patients developed grade 2 esophagitis in group 3 $(p=0.003)$. Under current Korean Health Insurance Policy, RT cost per person was $22.5 \%$ less in group 3 compared with others.

\section{Conclusion}

The current study demonstrated that 60 Gy in 15 fractions instead of 60 Gy in 20 fractions resulted in comparable clinical outcomes with excellent safety, direct cost saving, and improved convenience to the patients with tumors located at $\geq 1.5 \mathrm{~cm}$ from the esophagus.

Fax: 82-2-3410-2619

E-mail: ahnyc@skku.edu

Received October 17, 2015

Accepted March 2, 2016

Published Online March 9, 2016

Key words

*Won Kyung Cho and Jae Myoung Noh contributed equally to this work.

\section{Introduction}

Surgical resection has been the choice in treatment of patients with non-small cell lung cancer (NSCLC) with resectable primary tumor and without lymph node metastasis (cT1-3N0). Surgery alone usually can lead to 5-year survival rates of $60 \%-70 \%[1,2]$. However, some patients are not suitable for surgical resection because of several reasons, including inadequate cardio-pulmonary function, old age, medical comorbidities, and refusal of surgery by the patients. Definitive radiation therapy (RT) is considered a reasonable alternative to surgery in this clinical setting. The clinical outcomes following conventionally fractionated high dose RT delivering 60-66 Gy with 1.8 Gy or 2.0 Gy per fraction were generally rather unsatisfactory: 2-year local control (LC) rates were $55 \%-70 \%$; and 5 -year overall survival (OS) rates were $5 \%-42 \%$, respectively $[3,4]$.

As a way to intensify the local tumor control probability without compromising the risk of normal organ damage in 
high dose RT, a few altered fractionation schemes are considered [5]. Hyperfractionation, defined as more than one fraction per day at lower dose per fraction, is thought to be beneficial in suppressing tumor repopulation without increasing the risk of late morbidity. Accelerated fractionation, defined as more than one fraction per day without lowering the fractional dose, mainly aims to reduce tumor repopulation and to dramatically reduce the total treatment duration. Hyperfractionation and accelerated fractionation, however, are currently infrequently used in the routine clinics because of more frequent and severe acute toxicities in addition to the laboriousness and inconvenience both to the patients and the care-givers. Hypofractionation, defined as lower total fraction number at higher dose per fraction, is thought to be advantageous in controlling tumors that grow slowly and / or have a significant hypoxic fraction. Stereotactic body RT (SBRT), an extreme example of hypofractionation, is a reasonable alternative to surgery, but has a few practical limitations of tumor size and location associated with severe complication $[4,6]$.

The improvements both in diagnostics and RT techniques have enabled the delivery of high dose and conformal radiation to the target region only. However, coupled with these improved dose distribution profiles, the merits of shorter treatment duration seem to have been underestimated, mainly because of the traditional myth of late toxicity risk. The authors have employed a modest hypofractionation schedule in treatment of patients having NSCLC without lymph node metastasis, who are not ideal candidates for either surgery or SBRT for more than 15 years. Our clinical experiences with 60 patients, who had centrally located cT13N0 NSCLC and were treated with 54-60 Gy in 18-20 fractions by 3 Gy per fraction, were previously reported [7]. Soliman et al. [8] reported their promising clinical outcomes following 48-60 Gy in 12-15 fractions by 4 Gy per fraction in treating patients with peripherally located cT1-3N0 NSCLC. Encouraged and motivated by this, the authors have adopted a new fractionation schedule delivering 60 Gy in 15 fractions mainly to selected patients with non-centrally located tumors since 2011. The authors retrospectively assessed the clinical outcomes, toxicities, and cost-effectiveness following two modest hypofractionation schedules, under the consistent selection criteria in a single institute, and would report our experiences.

\section{Materials and Methods}

\section{Patients}

The current study was approved by the Institutional Review Board of Samsung Medical Center (2015-07-046), and the authors retrieved the data of the patients treated with definitive RT alone for NSCLC. Between January 2001 and June 2014, 131 patients underwent definitive RT alone for newly diagnosed cT1-3N0 NSCLC. Histopathologic confirmation of non-small cell carcinoma was achieved in all patients, and the clinical stages according to the seventh American Joint Committee on Cancer staging manual were assigned based on chest computed tomography (CT) in all and fluoro-deoxyglucose positron emission tomography (PET)-CT in 109 patients (87.9\%). In 17 patients (13.7\%), who had equivocal finding on the hilar and / or mediastinal lymph node involvement on imaging studies, endobronchial ultrasonographic aspiration biopsy was performed to confirm the absence of lymph node metastasis. After excluding four patients who did not complete RT and three who were lost to follow-up immediately following RT, 124 patients were included in the current analysis.

\section{Radiation therapy}

All patients underwent $\mathrm{CT}$ simulation typically in the supine position and the gross tumor volumes (GTVs) were delineated under lung window setting. The clinical target volumes (CTV) were generated with $5 \mathrm{~mm}$ expansion of the GTVs in all directions, which were then modified considering the adjacent anatomic limits. In principle, elective nodal irradiation (ENI) to the ipsilateral hilar and/or mediastinal lymphatics was not routinely added, however, was optionally added mainly to those with centrally located and large tumors. In 20 patients, who had primary tumors located in the lower lobes and/or showed significant respiratory motion, the GTVs were delineated on the internal target volume concept following four-dimensional CT. Three or four coplanar beams of 4-, 6-, or 10-MV photons from a linear accelerator were arranged so as to cover the CTVs plus $1.5 \mathrm{~cm}$ margins while avoiding the dose-limiting normal organs such as the spinal cord, lung, heart, and esophagus as much as possible. In patients who did not undergo four-dimensional CT, an extra $5 \mathrm{~mm}$ margin was added along the cranio-caudal direction. $\mathrm{V}_{20}$ of the lung was to be kept $\leq 27.5 \%$ and the mean lung dose was $\leq 16$ Gy [9]. Maximum doses to the spinal cord and esophagus were not to exceed $35 \mathrm{~Gy}$ and $45 \mathrm{~Gy}$, respectively. The prescription policy was to deliver at least $97 \%$ of the prescribed dose to $95 \%$ of the CTVs. 


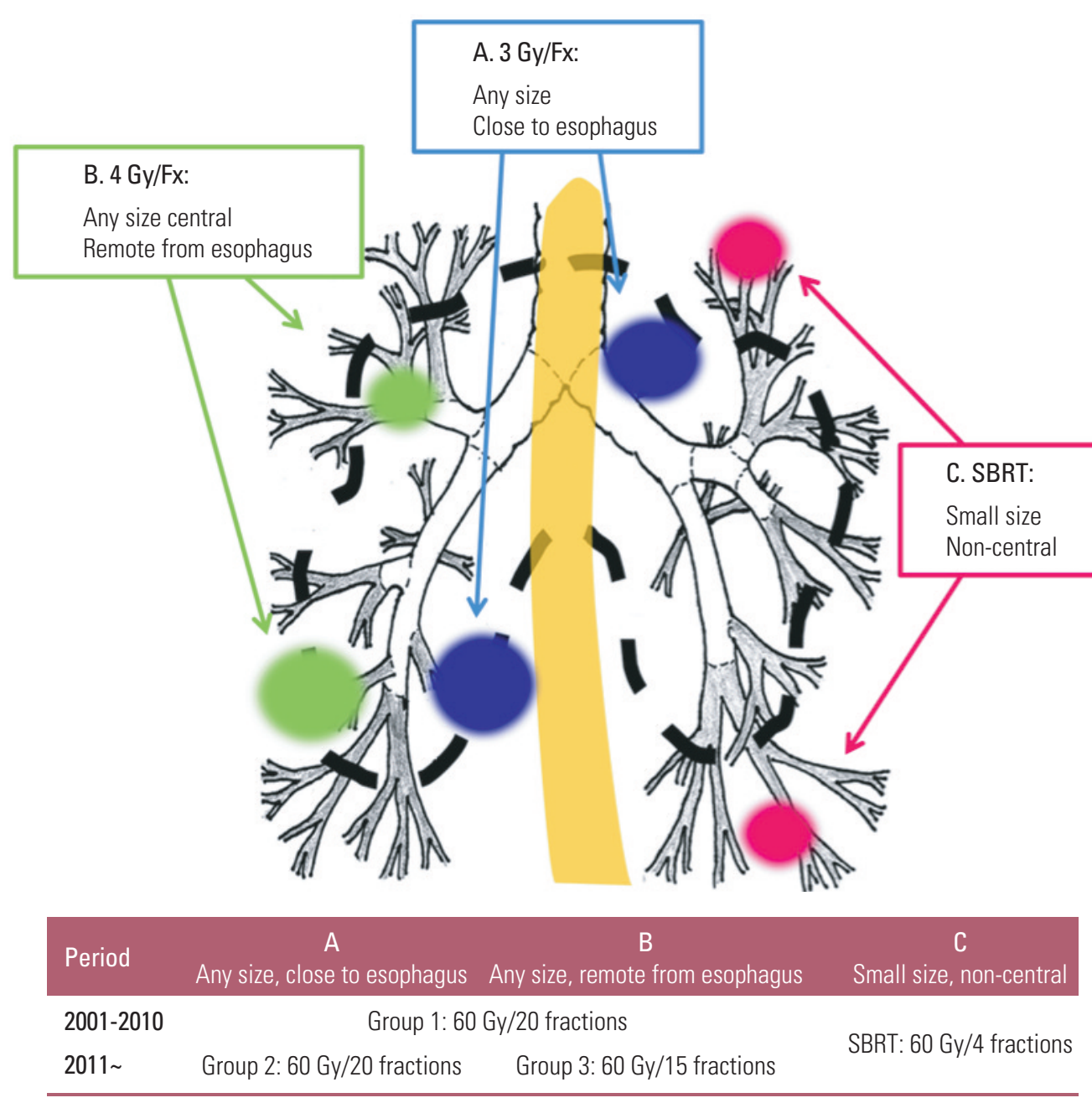

Fig. 1. Illustration of selection criteria of fractionation scheme in the current study. SBRT, stereotactic body radiation therapy.

From 2001 till 2010, one dose-fractionation schedule was used for delivery of 60 Gy in 20 fractions over 4 weeks to 79 patients (group 1). Since 2011 and thereafter, however, two different dose-fractionation schedules were used for delivery of $60 \mathrm{~Gy}$ in either 20 fractions over 4 weeks to 20 patients (group 2) or in 15 fractions over 3 weeks to 25 (group 3), respectively. The biologically equivalent doses at $\alpha / \beta$ of $10 \mathrm{~Gy}\left(\mathrm{BED}_{10}\right)$ were $78 \mathrm{~Gy}$ and $84 \mathrm{~Gy}$ to the patients in groups 1 and 2 and those in group 3, respectively. The selection of dose schedule in groups 2 and 3 depended on the location, size, and the geometry of the tumor in relation to the esophagus. If the shortest distance between the CTV margin and the esophagus was $\geq 1.5 \mathrm{~cm}, 60 \mathrm{~Gy}$ in 15 fractions was preferred to 60 Gy in 20 fractions (Fig. 1).

\section{Follow-up evaluation and statistical analysis}

Follow-up visits and imaging were obtained according to institutional protocol and included a follow-up visit with a chest CT scan 1 month after treatment and 3-4 months interval follow-up visit with either chest CT scan or PET scans alternatingly. Toxicity was graded using the Common Terminology Criteria for Adverse Events ver. 4.0.

OS, progression-free survival (PFS), and LC were calculated as the interval from the first date of treatment to the date of death or last follow-up, any progression detection, and consequent tumor growth at the primary site, respectively. Locoregional failure was defined as progression of primary tumor or regional lymphatics. Morbidities following RT and the cost incurred by RT course following two dose schemes were compared. 
Table 1. Patients' characteristics

\begin{tabular}{|c|c|c|c|c|c|}
\hline Variable & Total $(n=124)$ & Group $1(n=79)$ & Group $2(n=20)$ & Group $3(n=25)$ & p-value \\
\hline Age (yr) & $74(54-89)$ & $71(54-87)$ & $76.0(68-82)$ & $78(58-89)$ & 0.004 \\
\hline \multicolumn{6}{|l|}{ Sex } \\
\hline Male & $98(79.0)$ & $60(75.9)$ & $18(90.0)$ & $20(80.0)$ & 0.383 \\
\hline Female & $26(21.0)$ & $19(24.1)$ & $2(10.0)$ & $5(20.0)$ & \\
\hline \multicolumn{6}{|l|}{ Histologic type } \\
\hline Adenocarcinoma & $36(29.0)$ & $21(26.6)$ & $6(30.0)$ & $9(36.0)$ & 0.849 \\
\hline Squamous cell carcinoma & $71(57.3)$ & $46(58.2)$ & $11(55.0)$ & $14(56.0)$ & \\
\hline Others & $17(13.7)$ & $12(15.2)$ & $3(15.0)$ & $2(8.0)$ & \\
\hline $\mathrm{FEV}_{1}(\mathrm{~L})$ & $1.81(0.69-3.33)$ & $1.70(0.69-3.33)$ & $1.86(1.24-2.69)$ & $1.96(0.87-2.76)$ & 0.348 \\
\hline \multicolumn{6}{|l|}{ Performance status } \\
\hline ECOG 0-1 & $68(54.8)$ & $47(59.5)$ & $7(35.0)$ & $14(56.0)$ & 0.247 \\
\hline ECOG 2 & $51(41.1)$ & $28(35.4)$ & $12(60.0)$ & $11(44.0)$ & \\
\hline ECOG 3 & $5(4.0)$ & $4(5.1)$ & $1(5.0)$ & 0 & \\
\hline \multicolumn{6}{|l|}{ Reasons for no surgery } \\
\hline Poor pulmonary function & $62(50.0)$ & $43(54.4)$ & $8(40.0)$ & $11(48.0)$ & 0.537 \\
\hline Poor performance or old age & $35(28.2)$ & $19(24.1)$ & $9(45.0)$ & $7(28.0)$ & \\
\hline Comorbidity & $16(12.9)$ & $9(11.4)$ & $1(5.0)$ & $5(20.0)$ & \\
\hline Refusal of surgery & $11(8.9)$ & $8(10.1)$ & $2(10.0)$ & $1(4.0)$ & \\
\hline Tumor size $(\mathrm{cm})$ & $4.3(1.2-8.5)$ & $4.2(1.2-8.0)$ & $4.8(1.7-8.5)$ & $4.1(1.6-7.0)$ & 0.236 \\
\hline \multicolumn{6}{|l|}{ cT stage } \\
\hline cT1 & $25(20.2)$ & $15(19.0)$ & $3(15.0)$ & $7(28.0)$ & 0.173 \\
\hline cT2 & $65(52.4)$ & $42(53.2)$ & $8(40.0)$ & $15(60.0)$ & \\
\hline cT3 & $34(27.4)$ & $22(27.8)$ & $9(45.0)$ & $3(12.0)$ & \\
\hline \multicolumn{6}{|l|}{ Tumor location } \\
\hline Central & $81(65.3)$ & $62(78.5)$ & $12(60.0)$ & $7(28.0)$ & $<0.001$ \\
\hline Non-central & $43(34.7)$ & $17(21.5)$ & $8(40.0)$ & $18(72.0)$ & \\
\hline \multicolumn{6}{|l|}{ Elective nodal irradiation } \\
\hline Yes & $31(25.0)$ & $23(29.1)$ & $8(40.0)$ & 0 & 0.003 \\
\hline No & $93(75.0)$ & $56(70.9)$ & $12(60.0)$ & $25(100)$ & \\
\hline
\end{tabular}

Values are presented as median (range) or number (\%). $\mathrm{FEV}_{1}$, forced expiratory volume in 1 second; ECOG, Eastern Cooperative Oncology Group.

The distribution of categorical variables was analyzed using the chi-square test. The survival rates were calculated using the Kaplan-Meier method and compared using the logrank test for univariate analysis. Multivariate analysis was described with hazard ratios (HR) and their 95\% confidence interval $(\mathrm{CI})$ was derived from the Cox proportional hazards model. A p-value of 0.05 or less was considered statistically significant. SPSS Statistics ver. 20 (IBM Co., Armonk, NY) was used for the analysis.

\section{Results}

\section{Patients' characteristics}

The patients' characteristics according to the groups are summarized in Table 1. The median age of all patients was 74 years (range, 54 to 89 years) and over three-quarters (98 patients, 79.0\%) were male. Squamous cell carcinoma was more common in 71 patients $(57.3 \%)$ than adenocarcinoma in $36(29.0 \%)$ and other types in $17(13.7 \%)$. The performance status was Eastern Cooperative Oncology Group (ECOG) $0-1$ in 68 patients (54.8\%), ECOG 2 in $51(41.1 \%)$, and ECOG 3 in five (4.0\%), respectively. All patients received RT as an alternative to surgical resection because they either had poor pulmonary function in 62 patients $(50.0 \%)$, had poor per- 
Table 2. Failure patterns and clinical outcomes

\begin{tabular}{|c|c|c|c|c|c|}
\hline Variable & Total $(n=124)$ & Group $1(n=79)$ & Group $2(n=20)$ & Group $3(n=25)$ & p-value \\
\hline Follow-up, median (range, mo) & $16.7(1.2-119.5)$ & $23.1(1.2-119.5)$ & $12.6(3.1-45.6)$ & $14.2(4.1-45.6)$ & 0.063 \\
\hline Progression, $\mathrm{n}(\%)$ & $72(58.1)$ & $51(64.6)$ & $10(50.0)$ & $11(44.0)$ & 0.140 \\
\hline Locoregional & 35 & 26 & 5 & 4 & 0.570 \\
\hline Distant & 29 & 20 & 4 & 5 & \\
\hline Combined & 8 & 5 & 1 & 2 & \\
\hline Death, n (\%) & $66(53.2)$ & $59(74.7)$ & $4(20.0)$ & $3(12.0)$ & $<0.001$ \\
\hline Local control at 2 years $(\%)$ & 62.6 & 59.3 & 56.9 & 75.2 & 0.174 \\
\hline Progression-free survival at 2 years $(\%)$ & 39.1 & 36.1 & 34.6 & 51.6 & 0.280 \\
\hline Overall survival at 2 years $(\%)$ & 59.1 & 51.3 & 74.4 & 86.2 & 0.002 \\
\hline
\end{tabular}

formance status or old age in 35 (28.2\%), had medical comorbidity in $16(12.9 \%)$, or refused to undergo surgery in 10 $(8.9 \%)$, respectively. No significant differences in the distributions of sex, histologic type, median forced expiratory volume in 1 second $\left(\mathrm{FEV}_{1}\right)$, and ECOG performance status were observed among the groups. The median tumor size of all patients was $4.3 \mathrm{~cm}$ (range, 1.2 to $8.5 \mathrm{~cm}$ ), and group 2 patients tended to have larger tumors than those in groups 1 and 3 , but the difference was not statistically significant (4.2 cm vs. $4.8 \mathrm{~cm}$ vs. $4.1 \mathrm{~cm}, \mathrm{p}=0.236)$. cT stages in all patients were cT1 in 25 patients $(20.2 \%)$, cT2 in $65(52.4 \%)$, and cT3 in $34(27.4 \%)$, respectively, and group 3 patients tended to have cT1-2 tumors more frequently than other groups, which, however, was not statistically significant. The proportion of patients having a "central tumor," defined as a tumor located within $2 \mathrm{~cm}$ of the proximal bronchial tree, was $65.3 \%$ of all patients, which was significantly lower in group 3 (78.5\% vs. $60.0 \%$ vs. $28.0 \%$, p < 0.001$)$. The proportion of patients receiving ENI (median, $30 \mathrm{~Gy}$ in 10 fractions) was $25.0 \%$ of all patients, which also was significantly different among groups (29.1\% vs. $40.0 \%$ vs. $0 \%, \mathrm{p}=0.003)$. The mean and median shortest distances between the CTV margin and the esophagus were $3.0 \mathrm{~cm}$ and $2.0 \mathrm{~cm}$ (range, 0.6 to $9.0 \mathrm{~cm}$ ) in group 2 and $4.6 \mathrm{~cm}$ and $4.2 \mathrm{~cm}$ (range, 1.5 to $9.6 \mathrm{~cm})$ in group $3(\mathrm{p}=0.021$ and $\mathrm{p}=0.012)$, respectively.

\section{Clinical outcomes}

During the median follow-up period of 16.7 months (range, 1.2 to 119.5 months), 72 patients (58.1\%) experienced disease progression and $66(53.2 \%)$ died. The first progression presented as locoregional failure in 35 patients $(48.6 \%)$, distant metastasis in $29(40.3 \%)$, and synchronous combined failure in eight $(11.1 \%)$, respectively (Table 2 ). The rates of LC, PFS, and OS of all patients at 2 years were $62.6 \%, 39.1 \%$, and $59.1 \%$, respectively. The LC and PFS rates at 2 years were not significantly different among groups $(59.3 \%$ and $36.1 \%$ vs. $56.9 \%$ and $34.6 \%$ vs. $75.2 \%$ and $51.6 \%, \mathrm{p}=0.174$ and $\mathrm{p}=0.280$, respectively). The OS rate at 2 years was significantly better in group 3 compared with others $(51.3 \%$ vs. $74.4 \%$ vs. $86.2 \%, \mathrm{p}=0.002$ ).

Prognostic significance of several factors was analyzed by univariate analyses, which included treatment group, age, sex, histologic type, performance status, tumor size, cT stage, and tumor location (Table 3). Age, sex, and tumor location were not significant factors affecting any of the clinical outcomes (LC, PFS, and OS). The significantly favorable factors on LC were cT1-2 $(p=0.002)$ and ECOG performance status of $0-1(p=0.042)$, those on PFS were $c T 1-2(p<0.001)$, tumor size of $3 \mathrm{~cm}$ or smaller ( $\mathrm{p}=0.002)$, ECOG performance status $0-1(p=0.033)$, and non-adenocarcinoma histology $(\mathrm{p}=0.038)$, and those on OS were $c T 1-2(p=0.009)$ and treatment group 2 or $3(p=0.002)$, respectively. Tumor size of $3 \mathrm{~cm}$ or smaller was a favorable factor with borderline significance on OS $(\mathrm{p}=0.060)$.

In multivariate analyses, the significantly favorable factors on LC were cT1-2 $(p=0.014)$, those on PFS were cT1-2 $(p=0.002)$, non-adenocarcinoma histology $(p=0.003)$, and ECOG performance status of $0-1(\mathrm{p}=0.009)$, and those on OS were treatment group III $(\mathrm{p}=0.026)$ and $\mathrm{cT} 1-2(\mathrm{p}=0.035)$, respectively. cT1-2, as in univariate analyses, was the most powerful favorable factor affecting all three clinical outcomes of LC, PFS, and OS (HR, 0.363, 0.437, and 0.565; 95\% CI, 0.165 to $0.798,0.254$ to 0.752 , and 0.320 to 0.998 , respectively).

\section{Morbidity}

Eighty patients $(64.5 \%)$ developed radiation pneumonitis: most had grade 1 and 2 ( $42.7 \%$ and $17.7 \%$, respectively); four $(3.2 \%)$ had grade 3 ; and one $(0.8 \%)$ had grade 5 , respectively (Table 4). An 80-year-old female in group 1, who had a $2.6 \mathrm{~cm}$ tumor involving the left upper lobe and $\mathrm{FEV}_{1}$ value of $1.44 \mathrm{~L}$, developed symptomatic pneumonitis after 5 months of RT and died 7 months thereafter without evidence of cancer progression. No significant difference in the incidence of radiation pneumonitis was observed among the treatment 
Table 3. Prognostic factors by uni- and multi-variate analysis

\begin{tabular}{|c|c|c|c|c|c|c|c|c|c|c|}
\hline \multirow{2}{*}{ Variable } & \multirow{2}{*}{ No. } & \multirow{2}{*}{ LC $(\%)$} & \multicolumn{2}{|c|}{ p-value } & \multirow{2}{*}{ PFS $(\%)$} & \multicolumn{2}{|c|}{ p-value } & \multirow{2}{*}{ OS $(\%)$} & \multicolumn{2}{|c|}{ p-value } \\
\hline & & & Uni & Multi & & Uni & Multi & & Uni & Multi \\
\hline \multicolumn{11}{|l|}{ Treatment group } \\
\hline Group 1+2 & 99 & 59.6 & 0.206 & 0.541 & 36.2 & 0.279 & 0.615 & 54.3 & 0.009 & 0.026 \\
\hline Group 3 & 25 & 75.2 & & & 51.6 & & & 86.2 & & \\
\hline \multicolumn{11}{|l|}{ Age (yr) } \\
\hline$>75$ & 52 & 58.6 & 0.380 & 0.905 & 41.2 & 0.930 & 0.766 & 59.7 & 0.292 & 0.087 \\
\hline$\leq 75$ & 72 & 65.3 & & & 38.0 & & & 59.6 & & \\
\hline \multicolumn{11}{|l|}{ Sex } \\
\hline Male & 98 & 59.9 & 0.980 & 0.912 & 37.7 & 0.943 & 0.894 & 55.1 & 0.326 & 0.221 \\
\hline Female & 26 & 72.8 & & & 44.6 & & & 74.2 & & \\
\hline \multicolumn{11}{|l|}{ Histologic type } \\
\hline Adenocarcinoma & 36 & 68.6 & 0.527 & 0.253 & 32.6 & 0.038 & 0.003 & 47.0 & 0.289 & 0.035 \\
\hline Others & 88 & 61.4 & & & 42.2 & & & 63.6 & & \\
\hline \multicolumn{11}{|l|}{ Performance status } \\
\hline ECOG 0-1 & 68 & 70.4 & 0.420 & 0.013 & 46.9 & 0.033 & 0.009 & 63.7 & 0.166 & 0.201 \\
\hline ECOG 2-3 & 56 & 54.2 & & & 30.7 & & & 51.7 & & \\
\hline \multicolumn{11}{|l|}{ Tumor size $(\mathrm{cm})$} \\
\hline$\leq 3$ & 38 & 69.6 & 0.101 & - & 57.6 & 0.002 & - & 72.6 & 0.060 & - \\
\hline$>3$ & 86 & 58.7 & & & 30.4 & & & 52.3 & & \\
\hline \multicolumn{11}{|l|}{ cT stage } \\
\hline cT1-2 & 90 & 69.4 & 0.002 & 0.014 & 47.1 & $<0.001$ & 0.002 & 65.9 & 0.009 & 0.040 \\
\hline cT3 & 34 & 40.9 & & & 18.6 & & & 38.5 & & \\
\hline \multicolumn{11}{|l|}{ Tumor location } \\
\hline Central & 81 & 58.5 & 0.644 & 0.626 & 35.6 & 0.720 & 0.771 & 54.4 & 0.105 & 0.293 \\
\hline Non-central & 43 & 71.2 & & & 45.1 & & & 70.4 & & \\
\hline
\end{tabular}

LC, local control; PFS, progression-free survival; OS, overall survival; ECOG, Eastern Cooperative Oncology Group.

Table 4. Radiation therapy related morbidities

\begin{tabular}{|c|c|c|c|c|c|}
\hline Variable & Total $(n=124)$ & Group I (n=79) & Group II (n=20) & Group III (n=25) & p-value \\
\hline \multicolumn{6}{|c|}{ Pneumonitis } \\
\hline Grade 0 & $44(35.5)$ & $26(32.9)$ & $9(45.0)$ & $9(36.0)$ & 0.596 \\
\hline Grade 1 & $53(42.7)$ & $38(48.1)$ & $5(25.0)$ & $10(40.0)$ & \\
\hline Grade 2 & $22(17.7)$ & $11(13.9)$ & $6(30.0)$ & $5(20.0)$ & \\
\hline Grade 3 & $4(3.2)$ & $3(3.8)$ & 0 & $1(4.0)$ & \\
\hline Grade 4 & 0 & 0 & 0 & 0 & \\
\hline Grade 5 & $1(0.8)$ & $1(1.3)$ & 0 & 0 & \\
\hline \multicolumn{6}{|l|}{ Esophagitis } \\
\hline Grade 0 & $78(62.9)$ & $41(51.9)$ & $13(65.0)$ & $24(96.0)$ & 0.003 \\
\hline Grade 1 & $24(19.4)$ & $20(25.3)$ & $3(15.0)$ & $1(4.0)$ & \\
\hline Grade 2 & $22(17.7)$ & $18(22.8)$ & $4(20.0)$ & 0 & \\
\hline
\end{tabular}

Values are presented as number $(\%)$.

groups $(\mathrm{p}=0.596)$. Forty-six patients $(37.1 \%)$ developed radiation esophagitis: $24(19.4 \%)$ and $22(17.7 \%)$ had grade 1 and 2 , while no patients had grade 3 or higher. No patient in group 3 developed grade 2 esophagitis and this difference was statistically significant $(\mathrm{p}=0.003)$. 


\section{Treatment cost}

Under the Korean Health Insurance Policy as of August of 2015, the direct treatment costs incurred solely by RT course (including simulation and planning) per person were calculated as 3,380,000 Korean Won (KRW), approximately 2,860 USD, in groups 1 and 2 and 2,620,000 KRW, approximately 2,220 USD, in group 3, respectively.

\section{Discussion}

Hypofractionation is attractive in that dose escalation is possible while maintaining or shortening the treatment duration, and has recently been increasingly applied to a few solid tumor types including breast cancer, prostate cancer, and glioblastoma [10]. SBRT is an example of an extreme method of hypofractionation, which has emerged as a reasonable alternative to surgical resection in several clinical settings. SBRT has resulted in very promising LC rates approaching the $90 \%$ level when applied to NSCLC patients with early stage and operable lesions but are, at the same time, not physically fit for surgery [11]. Severe complications, however, have been reported in patients with centrally located tumors, typically within $2 \mathrm{~cm}$ from the central bronchial tree (2-year freedom from severe toxicity, 54\%) $[4,6]$. Thus centrally located tumors or large-sized tumors have usually been considered ineligible for SBRT due to fear of excessive toxicity risk. The optimal dose scheme for treatment of patients who are unfit for both surgical resection and SBRT has not yet been established.

Two recent prospective studies reported favorable clinical outcomes following high dose RT by modest hypofractionation schedules in patients who were unfit for both surgical resection and SBRT in CT1-2N0 NSCLC. The Cancer and Leukemia Group B (CALGB) trial 39904 prospectively evaluated accelerated three-dimensional conformal radiation therapy for delivery of 70 Gy in 17-29 fractions for early stage NSCLC and reported a median survival of 38.5 months and disease relapse rate of $25.6 \%$ [12]. A Canadian multi-institutional prospective phase II trial reported very promising 2-year LC rates of $87.4 \%$ following 60 Gy in 15 fractions [13]. The largest retrospective Canadian study reported a 2-year LC rate of $76.2 \%$ in 118 patients following a total dose of 48-60 Gy in 12-15 fractions, in which most patients received 48 Gy $(59.7 \%)$ or 52 Gy (37.1\%) [8]. Lee et al. [14] reported a 2-year LC rate of $74.6 \%$ in 26 patients following median 70 Gy (range, 60 to $72 \mathrm{~Gy}$ ) in 15-18 fractions. In the current study, the authors achieved a 2-year LC rate of $75.2 \%$ in group 3 following $60 \mathrm{~Gy}$ in 15 fractions, which was quite comparable with other studies mentioned above $[8,13,14]$. Actually, the 2-year LC rate following $60 \mathrm{~Gy}$ in 15 fractions as in the current study was numerically superior to those achieved by the authors following 60 Gy in 20 fractions: $56.9 \%$ in the current study (as in group 2); and $57.9 \%$ in a previous study [7]. Delivery of higher $\mathrm{BED}_{10}$ by $60 \mathrm{~Gy}$ in 15 fractions $\left(\mathrm{BED}_{10}, 84 \mathrm{~Gy}\right)$, compared with delivery of $60 \mathrm{~Gy}$ in 20 fractions $\left(\mathrm{BED}_{10}, 78 \mathrm{~Gy}\right)$, as in the current study, appeared to have been translated into numerically improved LC $(p=0.174)$ and PFS $(p=0.280)$, though without statistical significance, and significantly improved OS ( $\mathrm{p}=0.002)$, even though the patients' profiles were not the same (Table 2). Improved LC following 60 Gy in 15 fractions was also observed in patients with tumors larger than $3 \mathrm{~cm}(77.2 \%$ vs. $54.3 \%$, data not shown above). Two Canadian studies were consistent in that tumor size larger than $3 \mathrm{~cm}$ was a significantly adverse factor with respect to distant metastasis $[8,13]$, which was also affirmed in univariate analyses in the current study (Table 3).

In multivariate analyses, however, less advanced cT stage was proved as a significantly favorable factor with respect to LC, disease-free survival (DFS), and OS in the current study (Table 3). In the current study, although group 3 was a significantly favorable factor for OS in multivariate analysis, the actual significance should be further validated because of the retrospective nature, small sample size, and possibility of case selection bias. Severe toxicities including radiation pneumonitis and esophagitis were uncommon following high dose RT by 4 Gy per fraction, mainly because elective mediastinal irradiation in patients with cN0 stage was omitted in all studies $[8,14]$. The authors have positively affirmed the safety of this regimen through the current study, particularly with respect to radiation esophagitis (Table 4).

Although 4 Gy per fraction demonstrated improved LC compared with $3 \mathrm{~Gy}$ per fraction even in tumors larger than $3 \mathrm{~cm}, \mathrm{BED}_{10}$ was still lower than the recommendations by others, who proposed minimum $\mathrm{BED}_{10}$ over 100 Gy mainly in the SBRT setting $[14,15]$. In this context, determination of an optimal dose schedule at limited toxicity level in patients who are not good candidates for surgical resection or SBRT by virtue of large tumor size and / or tumors located close to the esophagus is still a challenging issue. The results of two ongoing clinical trials, in treating centrally located NSCLC, are awaited: Radiation Therapy Oncology Group 0813 (NCT00750269) is a dose-escalation study starting from 50 Gy in five fractions to determine the optimal dose; and the LungTech trial NSCLC (NCT01795521) is to evaluate efficiency and toxicity by 60 Gy in eight fractions.

The issue of cost-effectiveness does not seem to have been well addressed. One study on postoperative breast irradiation showed that a cost-minimization strategy could result in a $43 \%$ cost reduction in RT [16]. One should be cautious; 
however, in the instances when the benefit of cost-saving following hypofractionated RT is offset by greater cost in managing the subsequent toxicity, and this type of negative trade-off should not be justified. Though the fraction number was reduced from 20 to 15 in the current study, the authors achieved even better clinical outcome profiles which included the toxicity profiles at a reduced direct cost of $22.5 \%$ (3,380,000 KRW vs. 2,620,000 KRW). Assuming that roughly half of the RT alone candidates are to receive $60 \mathrm{~Gy}$ in 15 fractions, instead of 60 Gy in 20 fractions, the direct cost saving would reach approximately $12.2 \%$. Most Korean candidates for curative RT alone, instead of surgical resection or SBRT, usually require assistance in their daily lives either from family members or other care-givers, including escort to and from the RT facility on an out-patient basis. Though not measured herein, the overall cost saving, not to mention the patients' convenience, could become even greater, when considering the opportunity cost that could be incurred to patients and their helpers. In addition, more efficient use of $\mathrm{RT}$ resources may be realized, which is often critical and important in a busy clinic.

Compared to two previous prospective studies, the current study, though retrospective in nature, has a few strong points: the CALGB 39904 trial included tumors smaller than $4 \mathrm{~cm}$ and did not report clinical outcomes in detail based on the fractionation schemes; and Canadian phase II trial reported the outcomes following high dose RT by 4 Gy per fraction only without comparison with other fractionation schemes. The current study, under the consistent selection criteria and fractionation scheme, included patients with small to large sized tumors, and reported clinical outcomes, including the issue of cost-effectiveness, in detail based on two different fractionation schedules. As in other reports, patients with tumors larger than $3 \mathrm{~cm}$ had poor LC (2 year LC of $69.6 \%$ vs. $58.7 \%$ ), and among them, those treated with 60 Gy in 15 fractions had better LC and survival outcomes. This implies that larger tumors might require a higher dose for LC as previously suggested in many other reports. Effort to escalate radiation dose safely using a proton beam would be highly rewarding, even in the situation when tumors are closely located to critical normal structures, like the esophagus $[17,18]$.

Proper use of intensity-modulated radiotherapy (IMRT) or proton beam therapy could be advantageous in safely deliv- ering higher radiation dose to the target. Videtic et al. [19] reported excellent LC and favorable survival (3-year LC and OS were $94 \%$ and $52 \%$ ) with tolerable toxicity profiles in treatment of medically inoperable stage I NSCLC patients with $50 \mathrm{~Gy}$ in 10 fractions using the IMRT technique alone. Yamashita et al. [20] reported 92\% LC at 2 years following 48-56 Gy in 4-7 fractions in treatment of cT1-3 NSCLC patients using volumetric modulated arc therapy. Bush et al. [21] reported the clinical outcomes following hypofractionated proton beam therapy in treatment of patients with cT12N0 NSCLC: by escalating the dose from 60 Gy in 10 fractions to $70 \mathrm{~Gy}$ in 10 fractions, improved 4-year LC rates from $45 \%$ to $74 \%$ in cT2 tumors were achieved. Based on the excellent safety profiles, even in patients with large and centrally located tumors, hypofractionated proton beam therapy should be regarded as a promising alternative instead of photon beam therapy in treatment of early stage NSCLC patients who are not amenable to surgical resection or SBRT.

\section{Conclusion}

In summary, the current study demonstrated that 60 Gy in 15 fractions by 4 Gy per fraction resulted in comparable, even improved, clinical outcomes with excellent safety, particularly in patients with peripherally located cT1-3N0 NSCLC who are poor candidates for surgical resection or SBRT. By reducing the fraction number from 20 to 15 , in addition to the saving in direct and opportunity cost, improved patients' convenience, more efficient use of resources would be anticipated. To further improve the clinical outcomes, particularly in larger tumors, risk adaptive dose escalation using advanced RT techniques, such as IMRT or proton beam therapy, might be warranted.

\section{Conflicts of Interest}

Conflict of interest relevant to this article was not reported.

\section{References}

1. Adebonojo SA, Bowser AN, Moritz DM, Corcoran PC. Impact of revised stage classification of lung cancer on survival: a military experience. Chest. 1999;115:1507-13.
2. Naruke T, Goya T, Tsuchiya R, Suemasu K. Prognosis and survival in resected lung carcinoma based on the new international staging system. J Thorac Cardiovasc Surg. 1988;96:440-7. 
3. Rowell NP, Williams CJ. Radical radiotherapy for stage I/II non-small cell lung cancer in patients not sufficiently fit for or declining surgery (medically inoperable): a systematic review. Thorax. 2001;56:628-38.

4. Timmerman R, McGarry R, Yiannoutsos C, Papiez L, Tudor $\mathrm{K}$, DeLuca J, et al. Excessive toxicity when treating central tumors in a phase II study of stereotactic body radiation therapy for medically inoperable early-stage lung cancer. J Clin Oncol. 2006;24:4833-9.

5. Brenner DJ, Hall EJ. Fractionation and protraction for radiotherapy of prostate carcinoma. Int J Radiat Oncol Biol Phys. 1999;43:1095-101.

6. Corradetti MN, Haas AR, Rengan R. Central-airway necrosis after stereotactic body-radiation therapy. $\mathrm{N}$ Engl J Med. 2012;366:2327-9.

7. Oh D, Ahn YC, Kim B, Pyo H. Hypofractionated three-dimensional conformal radiation therapy alone for centrally located cT1-3N0 non-small-cell lung cancer. J Thorac Oncol. 2013;8: 624-9.

8. Soliman H, Cheung P, Yeung L, Poon I, Balogh J, Barbera L, et al. Accelerated hypofractionated radiotherapy for earlystage non-small-cell lung cancer: long-term results. Int J Radiat Oncol Biol Phys. 2011;79:459-65.

9. Oh D, Ahn YC, Park HC, Lim DH, Han Y. Prediction of radiation pneumonitis following high-dose thoracic radiation therapy by $3 \mathrm{~Gy}$ / fraction for non-small cell lung cancer: analysis of clinical and dosimetric factors. Jpn J Clin Oncol. 2009; 39:151-7.

10. Ray KJ, Sibson NR, Kiltie AE. Treatment of breast and prostate cancer by hypofractionated radiotherapy: potential risks and benefits. Clin Oncol (R Coll Radiol). 2015;27:420-6.

11. Oh D, Ahn YC, Seo JM, Shin EH, Park HC, Lim DH, et al. Potentially curative stereotactic body radiation therapy (SBRT) for single or oligometastasis to the lung. Acta Oncol. 2012;51: 596-602.

12. Bogart JA, Hodgson L, Seagren SL, Blackstock AW, Wang X, Lenox R, et al. Phase I study of accelerated conformal radiotherapy for stage I non-small-cell lung cancer in patients with pulmonary dysfunction: CALGB 39904. J Clin Oncol. 2010; 28:202-6.

13. Cheung P, Faria S, Ahmed S, Chabot P, Greenland J, Kurien
E, et al. Phase II study of accelerated hypofractionated threedimensional conformal radiotherapy for stage T1-3 N0 M0 non-small cell lung cancer: NCIC CTG BR.25. J Natl Cancer Inst. 2014;106:dju164.

14. Lee JH, Wu HG, Kim HJ, Park CI, Lee SH, Kim DW, et al. Hypofractionated three-dimensional conformal radiotherapy for medically inoperable early stage non-small-cell lung cancer. Radiat Oncol J. 2013;31:18-24.

15. Onishi H, Shirato H, Nagata Y, Hiraoka M, Fujino M, Gomi K, et al. Hypofractionated stereotactic radiotherapy (HypoFXSRT) for stage I non-small cell lung cancer: updated results of 257 patients in a Japanese multi-institutional study. J Thorac Oncol. 2007;2(7 Suppl 3):S94-100.

16. Greenup RA, Camp MS, Taghian AG, Buckley J, Coopey SB, Gadd M, et al. Cost comparison of radiation treatment options after lumpectomy for breast cancer. Ann Surg Oncol. 2012; 19:3275-81.

17. Koshy M, Malik R, Weichselbaum RR, Sher DJ. Increasing radiation therapy dose is associated with improved survival in patients undergoing stereotactic body radiation therapy for stage I non-small-cell lung cancer. Int J Radiat Oncol Biol Phys. 2015;91:344-50.

18. Park S, Urm S, Cho H. Analysis of biologically equivalent dose of stereotactic body radiotherapy for primary and metastatic lung tumors. Cancer Res Treat. 2014;46:403-10.

19. Videtic GM, Stephans K, Reddy C, Gajdos S, Kolar M, Clouser $\mathrm{E}$, et al. Intensity-modulated radiotherapy-based stereotactic body radiotherapy for medically inoperable early-stage lung cancer: excellent local control. Int J Radiat Oncol Biol Phys. 2010;77:344-9.

20. Yamashita H, Haga A, Takahashi W, Takenaka R, Imae T, Takenaka $S$, et al. Volumetric modulated arc therapy for lung stereotactic radiation therapy can achieve high local control rates. Radiat Oncol. 2014;9:243.

21. Bush DA, Cheek G, Zaheer S, Wallen J, Mirshahidi H, Katerelos A, et al. High-dose hypofractionated proton beam radiation therapy is safe and effective for central and peripheral early-stage non-small cell lung cancer: results of a 12-year experience at Loma Linda University Medical Center. Int J Radiat Oncol Biol Phys. 2013;86:964-8. 